\title{
Animal Science - Recent progress and future challenges
}

JD Oldham

SAC, Edinburgh, United Kingdom

Email: john.oldham@sac.ac.uk

Animal science spans a broad compass. It tends to be considered as the study of animals (nature, function, productivity etc) that are under the management of man in some way. This differentiates it from zoology, excludes wildlife and provides boundaries with at least some aspects of veterinary and biomedical sciences that can be rather indistinct. It covers both the understanding of animals as biological systems and the role of animals as components of broader systems ("Animals as systems; animals in systems'). By being allied to animals that are under management, I take it as implicit that any consideration of progress in animal science needs to include not only progress in intellectual understanding, but also progress in the utility of that understanding in practice. This does not deny the value of animal science to inform broader biological understanding.

There has been considerable scientific effort and increases in understanding both on animals themselves and on their roles in systems, although rather more in the former than the latter. Equally there are future challenges both at the level of the animal as a phenotype and for animals in systems of management. In each case the great challenge is not simply to understand the behaviour (in its broadest sense) of the animal or system, but to be able to predict it.

"Recent" is a word that is open to some interpretation. The rate at which some new techniques can help to generate data in research can make it appear that rates of progress are accelerating. 'Recent' progress in such areas might be considered to span small numbers of years. But a broader perspective is needed to recognise some of the major areas of progress.

Progress in any science is driven by a mixture of imagination to generate the ideas that are worth pursuing, and the acquisition of appropriate techniques to allow those ideas to be tested. Sir John Hammond was a fount of ideas on reproduction, growth and development in the inter-war years and through the 1950s. His insights and techniques led the way in practical improvements in reproduction and his concepts of growth and development have generally stood the tests of time. Like Hammond's waves of growth progress in animal science has been influenced by waves of technical development. The advent of chromatographic techniques in the early post-war years allowed access to rapid analytical methods that underpinned progress in nutrition and physiology especially. Isotopic tracing techniques gave access to more quantitative understanding of metabolism as well as facilitating analysis. More recently molecular techniques have opened avenues to understanding that were previously closed. And advances in computational and statistical techniques have not only supported the rapid delivery of other methods but have also allowed the realisation of ideas that, in some areas (perhaps especially genetics) were formed a significant time ago but were inaccessible for want of computational power.

Progress in genetics has helped to develop animals whose rates of productivity is dramatically increased although sometimes with negative associated consequences - which are now being corrected by adjustments to selection approaches. The promise of molecular approaches to replace more conventional quantitative methods has not been fully realised, although the prospects of using genome-wide selection are considerable. Understanding of nutrition and nutritional biochemistry has yielded rationing schemes that work tolerably well and allow some control over the qualities of products that animals produce. Our understanding of reproductive processes has grown substantially, but too often the application of new knowledge has been in the 'catch-up' mode, aiming to correct problems introduced by other 'advances'. Research on pregnancy and lactation for all mammalian species, including our own, has, though, been considerably advanced through the animal sciences. Our understanding of the processes of disease have been enabled through the animal as well as the veterinary and medical sciences, and increasingly methods of control can be expected to include broad-based approaches that will call on the products of a range of animal sciences. The welfare of animals, as sentient beings, is better appreciated and open to assessment and improvement. But the prediction of phenotypic expression (what will an animal actually do given a knowledge of its genotype and its environment?) is still an aspiration rather than a reality.

On broader fronts the impacts of animals on their environment are better understood now than they were. Management of waste to reduce pollution or of grazing to achieve biodiversity goals is more possible. Animals also influence the social, or operating environments. For example systems of animal management can be important for social cohesion and are important sources of work power or of equity.

The impacts of animals on the environment are a source of much current concern, though. Some of this relates to issues around climate change, others to the management of pollution and of managing biodiversity. Yet other concerns are to do with perceived negative contributions of animal products to the healthiness of the human diet. At the same time the growing size of the human population and its ability to afford more animal products in the global diet has, and is likely to continue, to increase the global demands for animals and their products.

This is the big driver that creates the grand challenges for animal science to address. The need is to produce animal products in appropriate abundance, that are beneficial (or at worst not detrimental) to the healthiness of our diets, that are produced with minimal waste (by efficient use of resources and minimisation of losses through disease and reproductive failure especially) no negative impacts on, and preferably benefits to, the environment and in socially acceptable ways. To achieve these ends, if such is possible, I would expect to see the next stages of progress in the animal sciences being more integrative and predictive. Synthesis of understanding to enable the prediction of functional behaviour of animals as systems and animals in systems (and the behaviour of those systems) as well as continuing lines of discovery to enable this to happen should be to the fore. A sharp eye to the outcome, and not simply to the understanding, is merited. Sir John Hammond was an outstanding scientist - but his goal was science to enable efficient animal production. We still need that. 